\title{
Las relaciones laborales influyen en la planificación académica de la Facultad de Ciencias Económicas
}

\section{de la Universidad de Tumbes, 2020}

Labor relations influence the academic planning of the Faculty of Economic Sciences of the University of Tumbes, 2020 As relações trabalhistas influenciam o planejamento acadêmico da Faculdade de Ciências Econômicas da Universidade de Tumbes, 2020

\section{ARTÍCULO GENERAL}

Juan Bances jumbaro1904@gmail.com Universidad Nacional de Tumbes
Oscar Sandoval Universidad Nacional de Tumbes
Isabel Ramirez Universidad Nacional de Tumbes

Recibido 01 de Febrero 2022 | Arbitrado y aceptado 01 de Febrero 2022 | Publicado en 04 Marzo 2022

\section{RESUMEN}

La investigación tiene como propósito determinar que la en la planificación académica es influenciada por un buen ambiente laboral en la Facultad de Ciencias Económicas de la Universidad Nacional de Tumbes. El tipo de investigación fue no experimental con diseño descriptivo correlacional. Se utilizó como técnica a la encuesta y al cuestionario como instrumento de recolección de datos, el mismo que fue aplicado a 55 docentes. Al analizar las variables de estudio, el $49 \%$ resalta que casi siempre participo en las reuniones del Departamento, un $47 \%$ expreso que casi siempre existe un buen ambiente de trabajo; un $48 \%$ indico que a casi siempre cuentan con la colaboración de los colegas de otros departamentos, asimismo, el $60 \%$ expresaron que casi siempre cuentan con el apoyo de colegas del mismo departamento, de la Facultad de Ciencias Económicas, en razón a ello existe una correlación directa moderada entre las relaciones y la planificación académica, debido a que el coeficiente de correlación de Pearson es de 0,575 y significativa (Sig. $0,000<0,05$ ). Pudiéndose determinar que sí existe influencia de las relaciones en la planificación académica, aceptado la hipótesis alternativa

Palabras Claves. Planificación académica, relaciones, identidad, clima institucional.

\section{ABSTRACT}

The purpose of the research is to determine that academic planning is influenced by a good work environment in the Faculty of Economic Sciences of the National University of Tumbes. The type of research was non-experimental with descriptive correlational design. The survey was used as a technique and the questionnaire as a data collection instrument, the same one that was applied to 55 teachers. When analyzing the study variables, $49 \%$ emphasize that they almost always participate in Department meetings, $47 \%$ express that there is almost always a good work environment; $48 \%$ indicated that they almost always have the collaboration of colleagues from other departments, likewise, $60 \%$ expressed that they almost always have the support of colleagues from the same department, from the Faculty of Economic Sciences, for this reason there is a moderate direct correlation between relationships and academic planning, because the Pearson correlation coefficient is 0.575 and significant (Sig. $0.000<0.05$ ). Being able to determine that there is an influence of relationships in academic planning, accepting the alternative hypothesis

Keywords. Academic planning, relationships, identity, institutional climate.

\section{RESUMO}

O objetivo da pesquisa é determinar se o planejamento acadêmico é influenciado por um bom ambiente de trabalho na Faculdade de Ciências Econômicas da Universidade Nacional de Tumbes. O tipo de pesquisa foi não experimental com desenho correlacional descritivo. Utilizou-se a pesquisa como técnica e o questionário como instrumento de coleta de dados, o mesmo que foi aplicado a 55 professores. Ao analisar as variáveis do estudo, 49\% destacam que quase sempre participam das reuniões do Departamento, $47 \%$ expressam que quase sempre há um bom ambiente de trabalho; $48 \%$ indicaram que quase sempre contam com a colaboração de colegas de outros departamentos, da mesma forma, $60 \%$ manifestaram que quase sempre contam com o apoio de colegas do mesmo departamento, da Faculdade de Ciências Económicas, por isso existe um moderado correlação entre relacionamentos e planejamento acadêmico, pois o coeficiente de correlação de Pearson é 0,575 e significativo (Sig. 0,000<0,05). Ser capaz de determinar que há uma influência das relações no planejamento acadêmico, aceitando a hipótese alternativa

Palavras chaves. Planejamento acadêmico, relacionamentos, identidade, clima institucional. 


\section{INTRODUCCION}

A nivel internacional el dinamismo científico y tecnológico de las últimas décadas, y los consiguientes cambios en los valores, impresionan diferentes aspectos en la estructura de la sociedad, exigiendo nuevas perspectivas y estrategias sociales. Estas mutaciones constantes, tales como el desarrollo de cultivos, el aumento de la población y la complejidad de las tareas trajeron la necesidad de la creación de organizaciones, con objetivos y estructuras bien definidas, que buscan satisfacer los diversos segmentos de la sociedad; es así que nuestra sociedad es una sociedad de organizaciones, en la que estamos educados y pasamos nuestras vidas trabajando dependiendo casi siempre de ella, por lo que las relaciones percibidas directa o indirectamente por los trabajadores que laboran en ese medio ambiente tiene repercusiones en el comportamiento laboral, sistema organizacional y comportamiento individual. Tal es así que estamos abocados a determinar la influencia de esta variable en la planificación académica de la Facultad de Ciencias Económicas de la Universidad Nacional de Tumbes.

Diferentes investigaciones han abordado el tema sobre las relaciones laborales; a nivel de las empresas, Pacheco (2017) encontró una influencia positiva entre el clima organizacional y el desempeño en las PYMES hoteleras de Riobamba. Para Bermúdez, J; Pedraza, A; Rincón, C.(2015), afirman que es el conjunto de factores tangibles -como la infraestructura, los recursos, entre otros- y factores intangibles -como los valores, las relaciones interpersonales, las situaciones de interacción, entre otros - que se viven a diario en la universidad. Mendoza (2011), mostró la relación entre el clima y el desempeño docente evaluado por los padres, Segredo (2013). El clima organizacional repercute en las motivaciones y el comportamiento que tienen los miembros de una organización, su origen está en la sociología, disciplina en la cual el concepto de organización dentro de la teoría de las relaciones humanas, enfatiza la importancia del hombre en su función del trabajo por su participación en un sistema social. Las organizaciones están compuestas de personas que viven en ambientes complejos y dinámicos, lo que genera comportamientos diversos que influyen en el funcionamiento de los sistemas, que se organizan en grupos y colectividades; el resultado de esta interacción media en el ambiente que se respira en la organización.

Su estudio es de vital importancia a causa del enorme impacto que tiene sobre el comportamiento de los miembros de una organización, constituyendo una especie de 
reflejo de la vida de la organización y las condiciones en las cuales los trabajadores se desenvuelven en el ámbito laboral. González, Manríquez, Venegas (2014).

\section{MATERIALES Y MÉTODO}

\subsection{Tipo y Diseño de estudio}

La presente investigación es de tipo no experimental porque no se manipula la variable, (Hernández, et al, 2010).

El diseño de investigación es descriptivo correlacional. Los estudios correlaciónales tienen como propósito medir el grado de relación que existe entre dos o más conceptos, categorías o variables (Hernández, et al, 2010).

\subsection{Población y muestra}

La población fue de 55 docentes de la Facultad de Ciencias Económicas.

Tabla 1. Población y muestra.

\begin{tabular}{ll}
\hline DEPENDENCIA & TOTAL \\
\hline Docentes del Departamento Académico de Contabilidad & 18 \\
Docentes del Departamento Académico de Administración & 13 \\
Docentes del Departamento Académico de Economía & 10 \\
$\begin{array}{l}\text { Docentes del Departamento Académico de Matemática e } \\
\text { Informática }\end{array}$ & 14 \\
\hline TOTAL & 55 \\
\hline
\end{tabular}

Fuente: Cuadro de asignación de personal de la UNTUMBES.

La muestra está conformada por los 55 docentes de la FCCE-UNTUMBES.

\subsection{Métodos, técnicas e instrumento de recolección de datos.}

Los métodos que se utilizaron fueron el deductivo e inductivo.

\section{Técnicas:}

Se utilizó la técnica de la encuesta, la misma que se aplicó al personal docente de la Facultad de Ciencias Económicas de la Universidad Nacional de Tumbes, como instrumento se hizo uso del cuestionario; obteniéndose información relacionados con la actividad académica como objetivo de la investigación.

Hernández, et al, (2010) establecen que: “El procedimiento de recojo de datos significa un proceso para el recojo de la información de acuerdo al planteamiento del problema científico. Los datos que se recolecten deben ser confiables, válidos y objetivos. En el plan se debe incluir las variables definidas operativamente, la muestra y los recursos 
de tiempo, de apoyo institucional y económico. La definición operativa de las variables es esencial para la inferencia estadística".

\section{Instrumento de Recolección de datos:}

El cuestionario se aplicó a 55 docentes de la Facultad de Ciencias Económicas de la UNTUMBES, efectuándose 20 preguntas para medir la variable relaciones y 24 preguntas para la variable planificación académica. Con éste instrumento se buscó medir la influencia de una variable sobre la otra.

Hernández, et al, (2010) establecen que "la principal función de la medición de datos es lograr la congruencia entre los datos reales y los datos conceptuales. Los primeros proporcionan la evidencia empírica y los segundos, la evidencia teórica. En toda investigación con enfoque cuantitativo se ejecuta una técnica para para medir las variables establecidas en la hipótesis. La medición es efectiva si la técnica de recojo de la información guarda congruencia con las variables que han sido definidas conceptualmente".

\section{Confiabilidad del Instrumento de Recolección de datos:}

Se trabajó con el alfa de Cronbach, calificando a ambos cuestionarios con excelente confiabilidad.

\section{Plan de Procesamiento y análisis de datos:}

Una vez aplicado los cuestionarios para medir las variables, los datos se ordenaron y posteriormente se procesaron mediante el software IBMSPSS23. En dicho software se trabajó la correlación de las variables y dimensiones utilizando el coeficiente de Pearson.

Posteriormente la información procesada se ha descrito en tablas, las mismas que fueron elaboradas con hoja de cálculo Microsoft Excel, en ellas se muestra información importante, relacionado con el análisis de los resultados, los mismos que han sido discutidos, arribando a las conclusiones y recomendaciones de la investigación.

La Facultad de Ciencias Económicas de la Universidad Nacional de Tumbes, cuenta con cuatro departamentos académicos (ver Tabla 2)

Tabla 2. Profesores en la Facultad de Ciencias Económicas

\begin{tabular}{ll}
\hline DEPENDENCIA & TOTAL \\
\hline Docentes del Departamento Académico de Contabilidad & 18 \\
Docentes del Departamento Académico de Administración & 13 \\
Docentes del Departamento Académico de Economía & 10
\end{tabular}


Docentes del Departamento Académico de Matemática e Informática 14

TOTAL: 55

Los profesores son la población para el estudio, a quienes se les aplicó los diferentes instrumentos de recolección de información, tales como:

La encuesta para obtener información sobre todos los aspectos relacionados con la actividad académica; el cuestionario con un total de 20 preguntas para medir la variable relaciones y 24 preguntas para variable planificación académica. Con el cuestionario se buscó medir la influencia de una variable sobre la otra.

\section{RESULTADOS Y DISCUSIÓN}

En la tabla 3; se muestra la correlación entre las variables relaciones y planificación académica de la Facultad de Ciencias Económicas de la Universidad Nacional de Tumbes; determinándose que sí existe influencia directa moderada y significativa entre ambas variables.

Tabla 3: Correlación entre las variables relaciones y planificación académica

\begin{tabular}{llcc}
\hline & Relaciones & $\begin{array}{c}\text { Planificación } \\
\text { académica }\end{array}$ \\
\hline Relaciones & $\begin{array}{l}\text { Coeficiente Pearson } \\
\text { Significancia } \\
\text { (bilateral)" }\end{array}$ & 1 &, 519 \\
& Muestra (N) & 55 &, 000 \\
Planificación & Coeficiente Pearson &, 519 & 55 \\
académica & $\begin{array}{l}\text { Significancia. } \\
\text { (bilateral)" }\end{array}$ &, 000 & 1 \\
& Muestra (N) & 55 & 55 \\
\hline
\end{tabular}

Producto de la correlación de Pearson se observa que el P-valor es $0,000<0,05$, por lo que se puede determinar que, sí existe influencia de la variable relaciones en la planificación académica de la Facultad de Ciencias Económicas de la Universidad Nacional de Tumbes, aceptando la hipótesis alternativa.

Los docentes manifestaron que a pesar del esfuerzo que brindan dentro y fuera del horario de trabajo no tienen ninguna recompensa, sin embargo, están siempre dispuestos a laborar a tiempo completo por la institución, lo cual significa una identificación con la institución. Dichos resultados concuerdan con lo manifestado por Ojeda y Ferrer (2010) 
quienes expresan que existe una alta relación de dependencia entre las variables, lo que puso en evidencia que la planificación académica es afectada por los elementos, categorías y tipos de relaciones imperantes en las instituciones básicas estudiadas.

Al analizar las variables de estudio, el $49 \%$ resalta que casi siempre participo en las reuniones del Departamento, un 47\% expreso que casi siempre existe un buen ambiente de trabajo; un 48\% indico que casi siempre cuentan con la colaboración de los colegas de otros departamentos, asimismo, el 60\% expresaron que casi siempre cuentan con el apoyo de colegas del mismo departamento, de la Facultad de Ciencias Económicas, en razón a ello existe una correlación directa moderada entre las relaciones y la planificación académica, debido a que el coeficiente de correlación de Pearson es de 0,575 y significativa (Sig. 0,000<0,05). (anexo $\mathrm{N}^{\circ} 01$ ).Pudiéndose determinar que sí existe influencia de las relaciones en la planificación académica, aceptado la hipótesis alternativa. En razón a ello Sevilla, Galaz y Arcos (2008) indican que la participación de las comunidades académicas en las tareas de planeación del quehacer universitario supone, por una parte, el reconocimiento del valor de la experiencia y el conocimiento de los académicos en los diversos aspectos de la dinámica institucional y que, en consecuencia, los productos concretos de la planeación pueden tener una mayor riqueza conceptual, asimismo Montoya, P. et al (2017) expresan que un mayor nivel de apoyo y confianza por parte de los superiores a los trabajadores, se vincula a un aumento de la satisfacción con el trabajo.

Se determinó que, si existe influencia del ambiente físico en la planificación académica de la Facultad de Ciencias Económicas de la Universidad Nacional de Tumbes (anexo $\mathrm{N}^{\circ}$ 02) debido a que el coeficiente de correlación de Pearson es 0,370 con un Pvalor de 0,005 menor al nivel de significancia establecido $(0,05)$. Los docentes manifestaron que a veces el espacio físico, las condiciones de temperatura, las condiciones de iluminación y el ruido les permiten desempeñar su trabajo con normalidad, dichos resultados concuerdan con lo manifestado por Pérez (2008), quién establece que los elementos de magnitud física presentes en el entorno laboral; calor, frio, ruido, concentración de oxígeno, vibraciones mecánicas, luz, radiaciones les deben permitir a los trabajadores desempeñar su trabajo con normalidad. Se determinó, asimismo que sí existe influencia de la identidad en la planificación académica de la Facultad de Ciencias Económicas de la Universidad Nacional de Tumbes, debido a que el coeficiente de correlación de Pearson es 0,330 con un P-valor de 0,014 menor al nivel de significancia establecido $(0,05)$, según lo indicado en (anexo $\mathrm{N}^{\circ} 03$ ). Los docentes manifestaron que 
casi siempre cumplen con los objetivos a pesar de los escasos recursos, y a veces la facultad innova y mejora para obtener el liderazgo y siempre están dispuestos a laborar a tiempo completo por la institución sintiéndose partícipes del proyecto de la Facultad.

\section{CONCLUSIONES}

Sí existe influencia directa moderada y significativa de las relaciones en la planificación académica de la Facultad de Ciencias Económicas de la Universidad Nacional de Tumbes.

Sí existe influencia de las relaciones en la planificación del currículo, de la carga académica y en la programación de horarios de la Facultad de Ciencias Económicas de la Universidad Nacional de Tumbes.

La planificación del currículo, el plan de estudios y los sílabos están diseñados de acuerdo a las exigencias de la normatividad vigente, contempla asignaturas acordes a las necesidades del mercado laboral, y es flexible para que los estudiantes puedan elegirlas.

Si existe influencia del ambiente físico en la planificación académica de la Facultad de Ciencias Económicas de la Universidad Nacional de Tumbes, y que a pesar de las condiciones del espacio físico, temperatura, iluminación y el ruido, esto no les impide desempeñar su trabajo con normalidad.

\section{Agradecimientos}

A los docentes de la Facultad de Ciencias Económicas de la Universidad Nacional de Tumbes por el apoyo con información para realizar esta investigación.

\section{Referencias Bibliográficas}

Bermúdez, J.; Pedraza, A.; Rincón, C. 2015. El clima organizacional en universidades de Bogotá desde la perspectiva de los estudiantes. Revista Electrónica de Investigación Educativa, 17(3): 1-12.

Cardona, D.; Zambrano, R. 2014. Revisión de instrumentos de evaluación de clima organizacional.

Disponible

en: https://www.sciencedirect.com/science/article/pii/S012359231400117X

Guzmán, R.; Pacheco, M. 2014. Comunicación familiar y desempeño académico en estudiantes universitarios Zona Próxima, Universidad del Norte Barranquilla, Colombia. Disponible en: https://www.redalyc.org/articulo.oa?id=85331022008. 20: 79-91 
Mendoza, A. 2011. Relación entre clima organizacional y desempeño docente en instituciones educativas de inicial de la red $\mathrm{N}^{\circ} 9$ - Callao. Tesis de Maestría. Universidad San Ignacio de Loyola. Disponible en: https://alicia.concytec.gob.pe/vufind/Record/USIL_041eea664e02d8afd72e2f3cbe ef5c49.

Montoya P.; Bello N.; Bermúdez N.; Burgos F.; Fuentealba M.; Padilla A. 2017. Satisfacción Laboral y su Relación con el Clima Organizacional en Funcionarios de una Universidad Estatal Chilena.19 (58): 7-13. Disponible en: https://scielo.conicyt.cl/pdf/cyt/v19n58/0718-2449-cyt-19-58-00007.pdf.

Pacheco, S. 2017. Modelo de clima organizacional y su relación con el desempeño laboral en las PYMES hoteleras de Riobamba. Tesis de Doctor. Universidad Nacional Mayor de San Marcos, Disponible en https://industrial.unmsm.edu.pe/upg/archivos/TESIS2018/DOCTORADO/tesis7.p $\underline{\mathrm{df}}$

Segredo P. 2013. Clima organizacional en la gestión del cambio para el desarrollo de la organización. Rev Cubana Salud Pública. 39 (2): 385-393; Disponible en: http://scielo.sld.cu/scielo.php?script=sci_arttext\&pid=S086434662013000200017.

Sevilla, J.; Galaz, J; Arcos, J. 2008. La participación del académico en procesos de planeación y su visión institucional. 2008. Revista electrónica de investigación educativa. 10 (2): 1-19.

Zambrano, J.; Angel, M.; Espinoza, E. 2017. Estudio sobre el clima organizacional en docentes de la universidad técnica de Machala. Revista Universidad y Sociedad. $9(2): 163-172$. 
7. Anexos.

$\operatorname{Anexo} \mathbf{N}^{\circ} 01$

Tabla 6: Correlación entre las relaciones y planificación académica.

\begin{tabular}{cccc}
\hline & Relaciones & $\begin{array}{c}\text { Planificación } \\
\text { académica }\end{array}$ \\
\hline \multirow{2}{*}{ Relaciones } & Coeficiente Pearson & 1 &, 575 \\
& Significancia (bilateral)" & &, 000 \\
Muestra (N) & 55 & 55 \\
Planificación & Coeficiente Pearson &, 575 & 1 \\
académica & Significancia. (bilateral)" &, 000 & \\
& Muestra (N) & 55 & 55 \\
\hline
\end{tabular}

Anexo $\mathbf{N}^{\circ} 02$

Tabla 5: Correlación entre el ambiente físico y planificación académica.

\begin{tabular}{|c|c|c|c|}
\hline & & $\begin{array}{l}\text { Ambiente } \\
\text { físico }\end{array}$ & $\begin{array}{c}\text { Planificación } \\
\text { académica }\end{array}$ \\
\hline \multirow{3}{*}{$\begin{array}{l}\text { Ambiente } \\
\text { físico }\end{array}$} & Coeficiente Pearson & 1 & ,370 \\
\hline & Significancia (bilateral)" & &, 005 \\
\hline & Muestra $(\mathrm{N})$ & 55 & 55 \\
\hline \multirow{3}{*}{$\begin{array}{l}\text { Planificación } \\
\text { académica }\end{array}$} & Coeficiente Pearson & ,370 & 1 \\
\hline & Significancia. (bilateral)" & 005 & \\
\hline & Muestra $(\mathrm{N})$ & 55 & 55 \\
\hline
\end{tabular}

\section{Anexo $\mathbf{N}^{\circ} 03$}

Tabla 8: Correlación entre la identidad y planificación académica.

\begin{tabular}{cccc}
\hline & Identidad & $\begin{array}{c}\text { Planificación } \\
\text { académica }\end{array}$ \\
\hline \multirow{2}{*}{ Identidad } & $\begin{array}{c}\text { Coeficiente Pearson } \\
\text { Significancia (bilateral)" }\end{array}$ & 1 &, 330 \\
& Muestra (N) & 55 &, 014 \\
Planificación & Coeficiente Pearson &, 330 & 55 \\
académica & $\begin{array}{c}\text { Significancia. (bilateral)" } \\
\text { Muestra (N) }\end{array}$ &, 014 & 1 \\
\hline
\end{tabular}

